\title{
Analysing Enthymematic Dialogue
}

In the previous chapter we sketched a picture of the role enthymemes and topoi play for dialogue updates. In this chapter we will move on to consider how the DGB is updated. In a dialogue model, situations where enthymematic reasoning occurs are characterised by the sequence of update rules that is required to bring about the progression of the dialogue. In addition to update rules, we also assume an update algorithm that controls in which order rules may be applied. However, we will not go into this in detail, but instead in the rules define the conditions for when an agent is licensed to apply that rule.

We will look at some examples and analyse them gradually introducing new concepts and describing for each update how the relevant features of the gameboard and the necessary update rules work. This way we hope to make clear how we perceive the various features of the gameboard in terms of types, while elucidating some points regarding the dynamics of enthymemes and topoi in this kind of interaction.

\subsection{Enthymeme Elicited by Why?}

On a rhetorical view of dialogue, the topoi in the resources of an agent may be drawn on to invent and interpret different kinds of enthymemes. When we talk about how things are, we sometimes use arguments to back up our claims or to give sufficient explanations for the state of affairs reported in our assertions.

In (4.1) we have an example of an interaction where an assertion is backed up with a premise so that the two moves by speaker $A$ make up an enthymematic argument. The premise of the enthymeme is elicited by the why-question in (4.1b). In this way the argumentative structure is made explicit-Sam is in hospital because he is sick. (4.1) is a slightly altered version of an example from the British National Corpus discussed and analysed by Schlöder et al. (2016), who argue that a why-question following an assertion is likely to elicit a reason for the content expressed in the first utterance being the case, rather than a reason for making the statement. In many contexts, if the relevance of an utterance is not clear, it is more intuitive to ask "why are you saying that?", "what do you mean?" or similar, rather than a bare "why?". However, there are exceptions 
to this, see for example Gregoromichelaki and Kempson (2015). Regardless of whether a why-question is factive (addresses the facts of a proposition made) or meta-communicative (addresses an interlocutor's reason for saying something), the reply to the why-question must evoke a recognisable topos. If it does not, there is no warrant for the response as a support or explanation of the statement preceding the why-question.
(4.1) a. A:Sam's in hospital.
b. $B$ : Why?
c. A: He's sick.

In our analysis of (4.1) we omit several steps that would be necessary if we were modelling this dialogue for example for the purpose of implementation. Since we are mostly concerned with the rhetorical aspects of the dialogue, we ignore at this point the machinery adding new move types to the agenda and other updates pertaining to the private field of the DGB. We will start at the point just after $A$ has uttered (4.1a), and the utterance "Sam's in hospital" is thus already integrated as the latest move on the DGB. We will refer to the type of the shared information state when (4.1a) has just been integrated as $T_{\text {initial }}$.

(4.2) $T_{\text {initial }} \sqsubseteq \operatorname{sh}:\left[\begin{array}{l}\operatorname{l-m}:\left[\begin{array}{l}\mathrm{x}=A: \text { Ind } \\ \mathrm{y}=B: \text { Ind } \\ \mathrm{ctnt}=\left[\begin{array}{l}\mathrm{x}=\operatorname{Sam}: \text { Ind } \\ \mathrm{c}_{\mathrm{h}}: \text { in_hospital }(\mathrm{x})\end{array}\right]: \text { RecType }\end{array}\right]\end{array}\right]$.

Since an information state potentially involves a wide range of information, and the types that we manipulate do not necessarily include all of this information, types of actual information states such as $T_{\text {initial }}$ involves at least as many constraints as the record types we take as our point of departure when updating the DGB. Thus $T_{\text {initial }}$ is a subtype of the record type to the right in (4.3). It is underspecified in terms of the fields present, for example, it lacks all private features of the DGB. Also, the previous move made in the interaction is not specified in terms of the content of that move, and the 'qud'-field does not provide any information about any questions already under discussion in the dialogue. We will use a dot notation to refer to paths in record types. For example, if $s: T_{\text {initial }}$, we can refer to "Sam" in (4.3) using the notation 's.sh.lm.e.ctnt.x'. 
In (4.2) the label 'sh' (for 'shared') is associated with a record type including the labels ' l-m', representing the latest moves, and 'qud', representing questions under discussion. (For reasons of space we will replace the label 'shared' with 'sh' and 'private' with 'pr' where necessary.)

Since we do not know anything about the conversation prior to this excerpt, we are, at this initial state, agnostic about any previous moves. We say that the label 'qud' is associated with the type "list of questions", and the label 'l-m' with a record type with the fields 'prev' and 'e', where 'prev' contains information about previous moves. How many previous moves should be included on the $\mathrm{DGB}$, that is, how many turns are in fact accessible to a dialogue participant is an empirical question. However, for the short dialogues we are considering here there is no need to limit the number of previous moves. In each type representing an information state, 'shared.l-m.e' contains information about the very latest move made.

\subsubsection{Integrate Why?}

Our first update rule thus concerns the update of $B$ 's information state where a why-question is pushed onto 'qud' given the assertion "Sam's in hospital" in (4.1a).

Now $B$ may choose to move on in the conversation accepting that Sam is in hospital or to address $A$ 's assertion for example by questioning it or by asking a follow up question. Let us assume now that $B$ intends to make a factive why-question investigating the reason for Sam being in hospital (the reasoning behind this intention we will leave aside for the time being). Following Schlöder et al. (2016), we will think of why-question as requests for reasons.

On a rhetorical view, an acceptable reason for Sam being in hospital would be one warranted by a recognisable topos. This is the case even if the whyquestion is not meant to inquire into the validity of the claim, but rather to find out the reason for Sam being in hospital. If the reply to the question would be something like "He is such a nice guy", we may speculate that the answer would not be accepted. The reason for this is that there is probably no topos readily available to any of the participants in this dialogue to be recognised as underpinning for the enthymeme "Sam is in hospital because he is such a nice guy". However, we could imagine contexts where the utterance would make perfect sense; there might be a story about Sam having intervened in a fight, since he is a nice guy, and therefore ended up having to undergo hospital treatment. However, on this scenario, the reply to the first question would probably be followed up by yet another question inquiring into why Sam being a nice guy is a reason for his being in hospital. 
It is important to emphasise that it is certainly not possible for a linguistic theory to predict which topoi are acknowledged or acceptable within a community or to a specific individual. However, it is possible to predict how reasoning in a particular situation may play out given the agents' access to a particular set of topoi.

In more technical terms, an enthymeme can be regarded as a function from a record of a particular type (corresponding to the antecedent of the enthymeme, in this case "Sam is sick") to a type corresponding to the consequent of the enthymeme, in this case "Sam is in hospital". A why-question is obtained by abstracting over $T_{\text {antecedent }}$ yielding a function from an antecedent type to an enthymeme. We see such a function in (4.3).

\section{(4.3) $\lambda t:$ Type $\cdot \lambda r: t \cdot T_{\text {consequent }}$}

When provided with an answer to the question, we may apply this function to a type $T_{\text {antecedent }}$ (corresponding to the answer to the why-question):

$$
\text { (4.4) } \lambda t: \text { Type } \cdot \lambda r: t \cdot T_{\text {consequent }}\left(T_{\text {antecedent }}\right)=\lambda r: T_{\text {antecedent }} \cdot T_{\text {consequent }}
$$

At the point in the dialogue when $B$ has uttered the why-question, we need an update function which allows $A$ to integrate the question on her DGB. This includes interpreting the question, that is, forming a hypothesis of what aspect of the previous utterance the "why?" relates to. This function must take an information state which, like (4.2), meets the requirement of having an assertion on 'shared.l-m.e', and update it so that the updated latest move is the why-question, while also pushing the same question on 'qud'. We refer to this function as $f_{\text {why_assert }}$. 


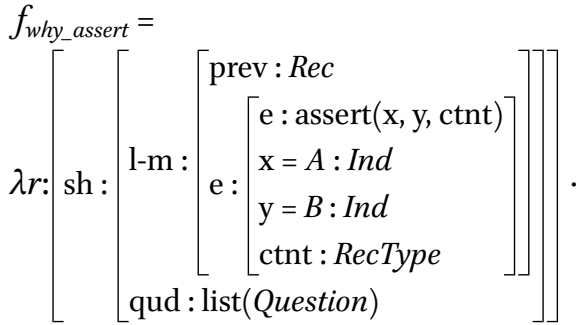

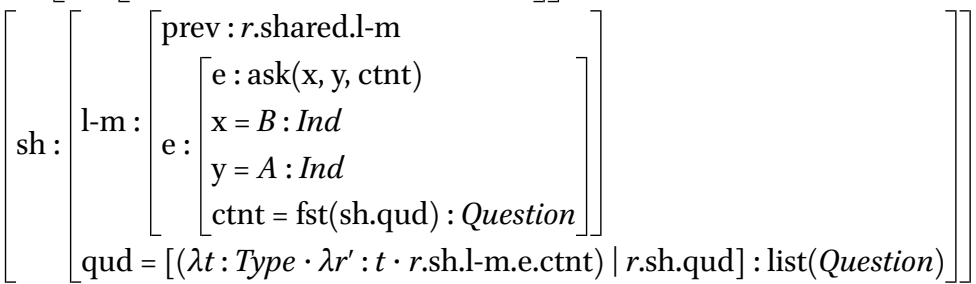

In (4.5) we see the function $f_{\text {why_assert }}$. It can be applied to an information state of the type where the very latest move, 'sh.l-m.e', is associated with a move of a type where an individual $A$ asserts something to an individual $B$.

In the result type of the function the label 'l-m' is associated with a type where the move type that was on 'l-m.e' in the previous information state is now on 'lm.prev', and the label ' $\mathrm{e}$ ' is associated with a why-question regarding the content of the previous move.

In (4.5) we also observe some list operators, namely '|' and 'fst', which is a function from a list to the first element of that list. Thus 'fst(sh.qud)' represents the first element on the list of questions on 'sh.qud'. The "pipe" (or "cons") operator, '", takes an element and a list and puts that element first in the list.

$f_{\text {why_assert }}$ can be applied to a record of the type in (4.2). However, the result of this operation includes the relevant updates brought about by the function application, but not any information about other dialogue features that might be present in the original information state (in this case of type $T_{\text {initial }}$ ). For example, $T_{\text {initial }}$ could include information about previous moves, other questions that have not yet been resolved, commitments, etc. In order to incorporate the new information with the old we need to asymmetrically merge $T_{\text {initial }}$ and the type obtained through the application of $f_{\text {why_assert }}$ to an information state of type $T_{\text {initial }}$.

\subsubsection{Asymmetric Merge}

As explained above, in order to obtain the type of the updated information state, the result of applying an update rule will have to be combined with the type of the initial information state. Cooper (2016) refers to this operation as asymmetric merge. For types which are not record types, for example ptypes, 
the asymmetric merge of two types $T_{1} \wedge T_{2}$ results in $T_{2}$. This means that for record types, any label that occurs in both $T_{1}$ and $T_{2}$ will after an asymmetric merge be associated with the type which that label is associated with in $T_{2}$. Labels which occur only in one of the record types will be associated with the same type in $T_{1} \wedge T_{2}$ as in one of the original types $T_{1}$ or $T_{2}$. If we look at our example, the type we get when we apply the function $f_{\text {why_assert }}$ to the initial information state, $s_{\text {inital }}$, is a type $T^{\prime}$, which has the same fields as the result (range) type of the function $f_{\text {why_assert }}$. However, this type is not necessarily the type of the updated information state since the type of $s_{\text {initial }}$ may have had more information in it.

To obtain the type of our updated information state, we must combine the type of the information state at the start of the update, $T_{\text {initial }}$ and the result type of the function application, $T^{\prime}$, so that everything that is on the gameboard before the update carries over to the new information state. In (4.6) we see the asymmetric merge of $T_{\text {initial }}$ and $T^{\prime}$, resulting in the type of $A$ 's information state after the application of rule $f_{\text {why_assert }}$ to $s_{\text {initial }}$ (where $s_{\text {initial }}$.sh.qud=rest), that is, the type of $A$ 's information state when she has integrated the why-question. 

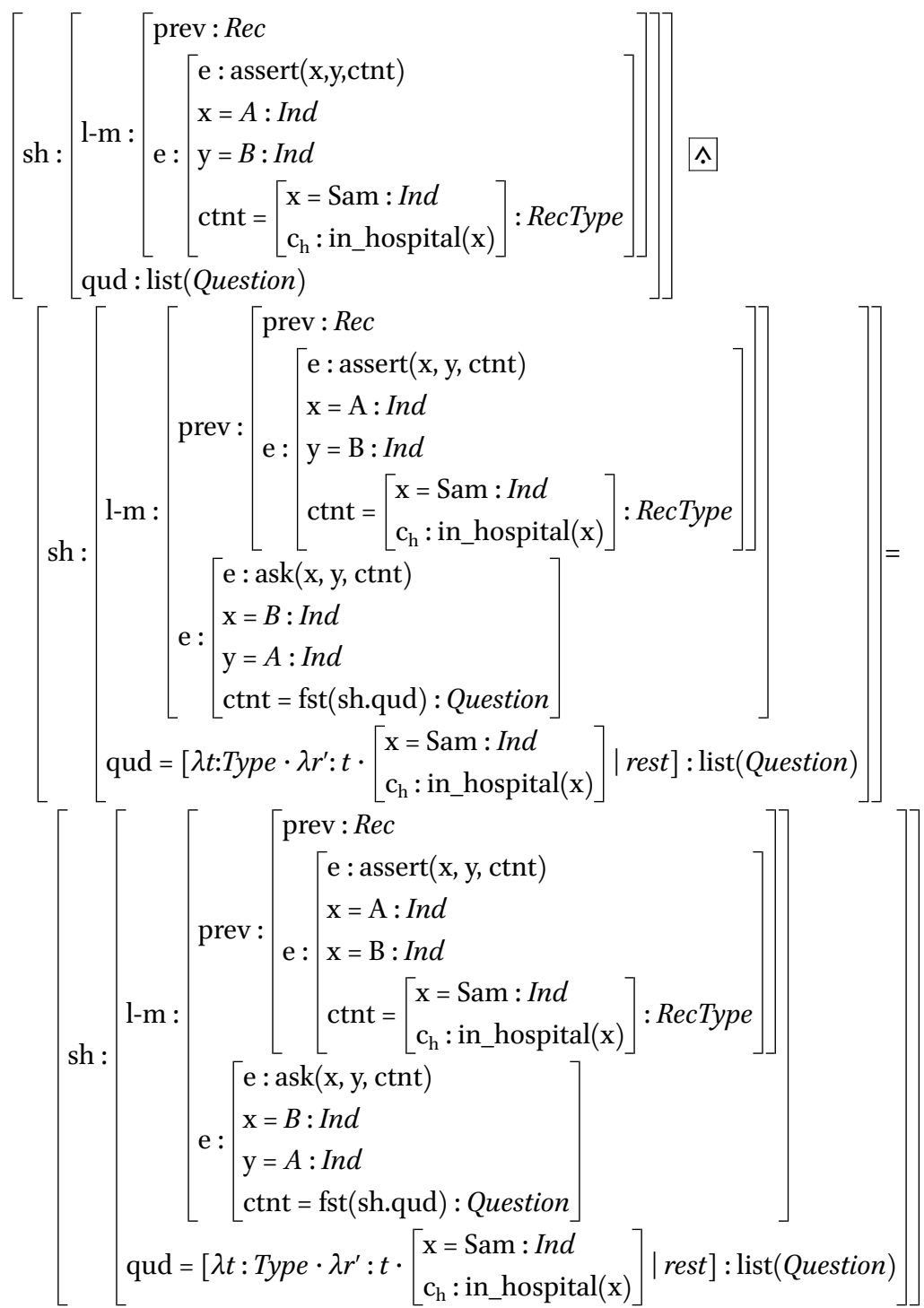

\subsubsection{Integrate Enthymeme ${ }_{\text {Why? }}$}

After having interpreted $B$ 's utterance “why?" in (4.1b), in order to reply, $A$ must decide what kind of answer to give. For now we will not discuss the reasoning behind this answer, and skip straight to the point where $B$ is interpreting and evaluating it. We will do this in steps starting at a point when $A$ 's reply in (4.1c) is integrated on 'l-m.e' on the DGB. Since the rhetorical structure of the reply in relation to the initial assertion is made explicit by the why-question, an enthymeme under discussion is also integrated on the shared DGB. $B$ then 
checks his resources for a topos warranting the enthymeme, and accommodates this topos. We will discuss accommodation of topoi further in section 4.2.5.

Let us now consider the type of $B$ 's information state just after $A$ has uttered (4.1c), as seen in (4.7). Let us call this type $T_{3}$.

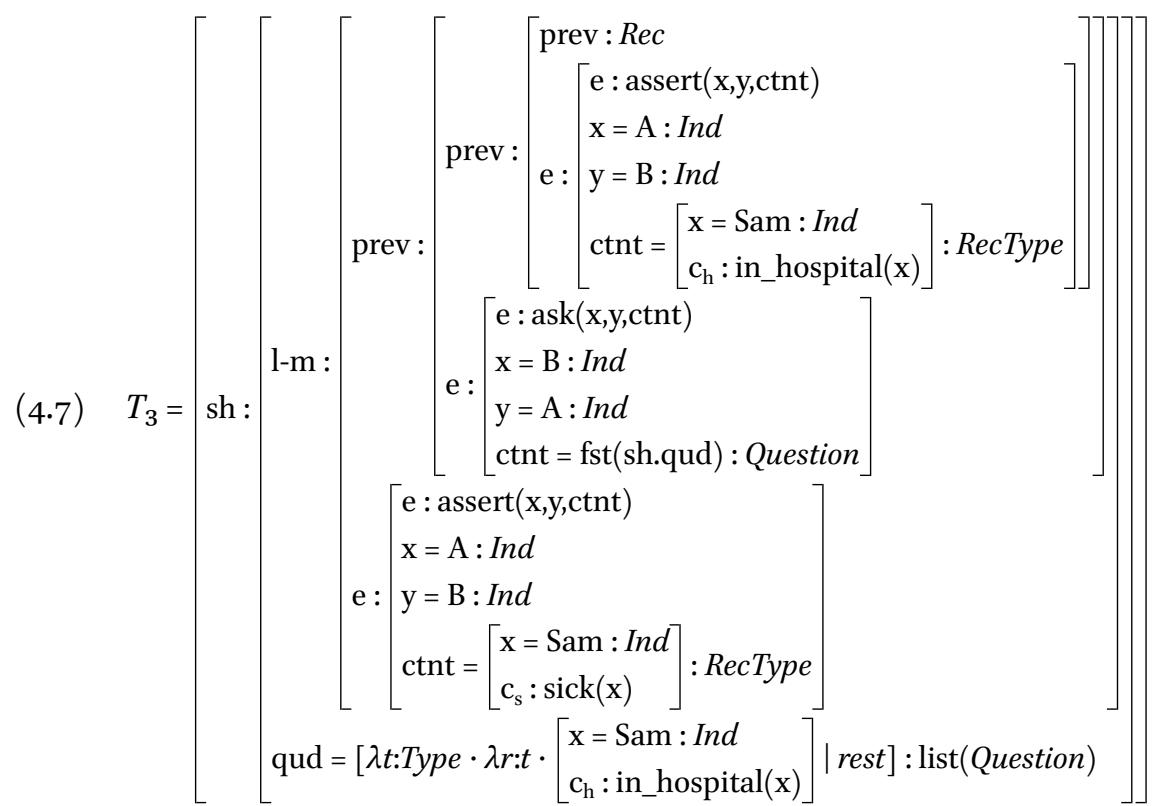

Now, as we discussed in the context of (4.4), an enthymeme elicited by a whyquestion can be considered as the result of applying the question to its answer. In this case the enthymeme under discussion is the why-question applied to the assertion "Sam is sick", as seen in (4.8).

$$
\begin{gathered}
\left.\lambda t: \text { Type } \cdot \lambda r: t \cdot\left[\begin{array}{l}
\mathrm{x}=\operatorname{Sam}: \text { Ind } \\
\mathrm{c}_{\mathrm{h}}: \text { in_hospital }(\mathrm{x})
\end{array}\right]\left(\begin{array}{l}
\mathrm{x}=\operatorname{Sam}: \text { Ind } \\
\mathrm{c}_{\mathrm{s}}: \operatorname{sick}(\mathrm{x})
\end{array}\right]\right)= \\
\lambda r:\left[\begin{array}{l}
\mathrm{x}=\operatorname{Sam}: \text { Ind } \\
\mathrm{c}_{\mathrm{s}}: \operatorname{sick}(\mathrm{x})
\end{array}\right] \cdot\left[\begin{array}{l}
\mathrm{x}=\operatorname{Sam}: \text { Ind } \\
\mathrm{c}_{\mathrm{h}}: \operatorname{in} \_ \text {hospital }(\mathrm{x})
\end{array}\right]
\end{gathered}
$$

A function for integrating an enthymeme after a factive why-question takes an information state where the third-to-latest move ('sh.l-m.prev.prev') and the latest move ('sh.l-m.prev') are assertions, and the question under discussion is a why-question, and returns a type of state where there is an enthymeme on 'eud' related to the question under discussion. We see this rule below in (4.9). 
(4.9) $\quad f_{\text {integrate_enthymeme_why }}=$

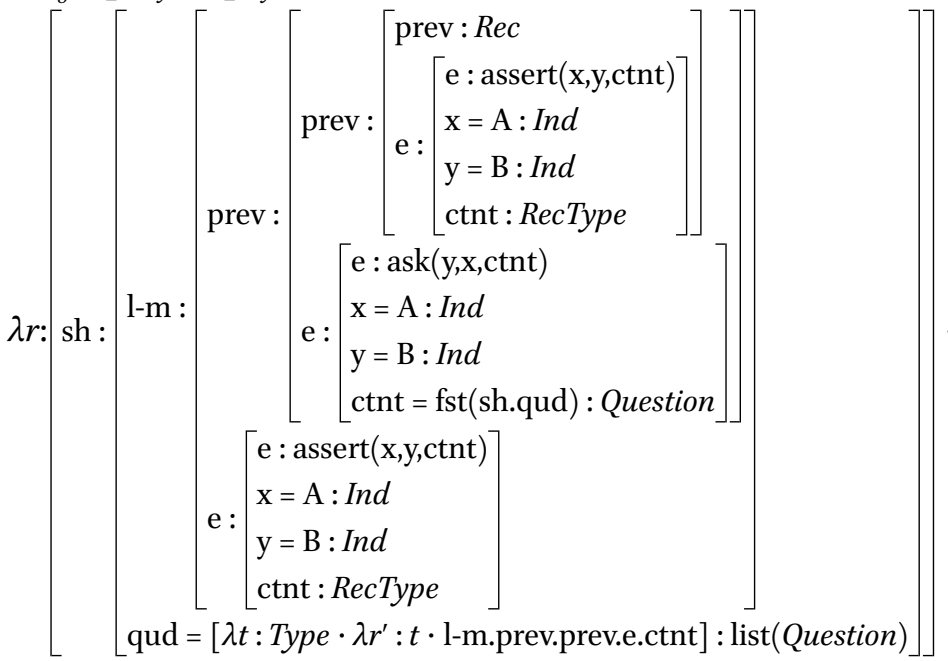

$$
\begin{aligned}
& {[\mathrm{sh}:[\text { eud }=[\text { r.sh.qud }(\text { r.sh.l-m.e.ctnt })]: \operatorname{list}(\text { Enthymeme })]]}
\end{aligned}
$$

We will now consider the result of applying $f_{\text {integrate_enthymeme_why }}$ to a record $s_{3}$ of type $T_{3}$ (shown in 4.10).

$$
\begin{aligned}
& \text { (4.10) } \left.\left.\begin{array}{l}
\text { integrate_enthymeme_why }\left(s_{3}\right)= \\
{\left[\operatorname{sh}:\left[\operatorname{eud}=\left[\lambda r:\left[\begin{array}{l}
\mathrm{x}=\operatorname{Sam}: \text { Ind } \\
\mathrm{c}_{\mathrm{s}}: \operatorname{sick}(\mathrm{x})
\end{array}\right] \cdot\left[\begin{array}{l}
\mathrm{x}=\operatorname{Sam}: \text { Ind } \\
\mathrm{c}_{\mathrm{h}}: \text { in_hospital }(\mathrm{x})
\end{array}\right]\right]: \operatorname{list}(\text { Enthymeme })\right.\right.}
\end{array}\right]\right]
\end{aligned}
$$

In (4.11) we see the type of $B$ 's information state after the application of $f_{\text {integrate_enthymeme_why }}$ and an asymmetric merge with $T_{3}$. 


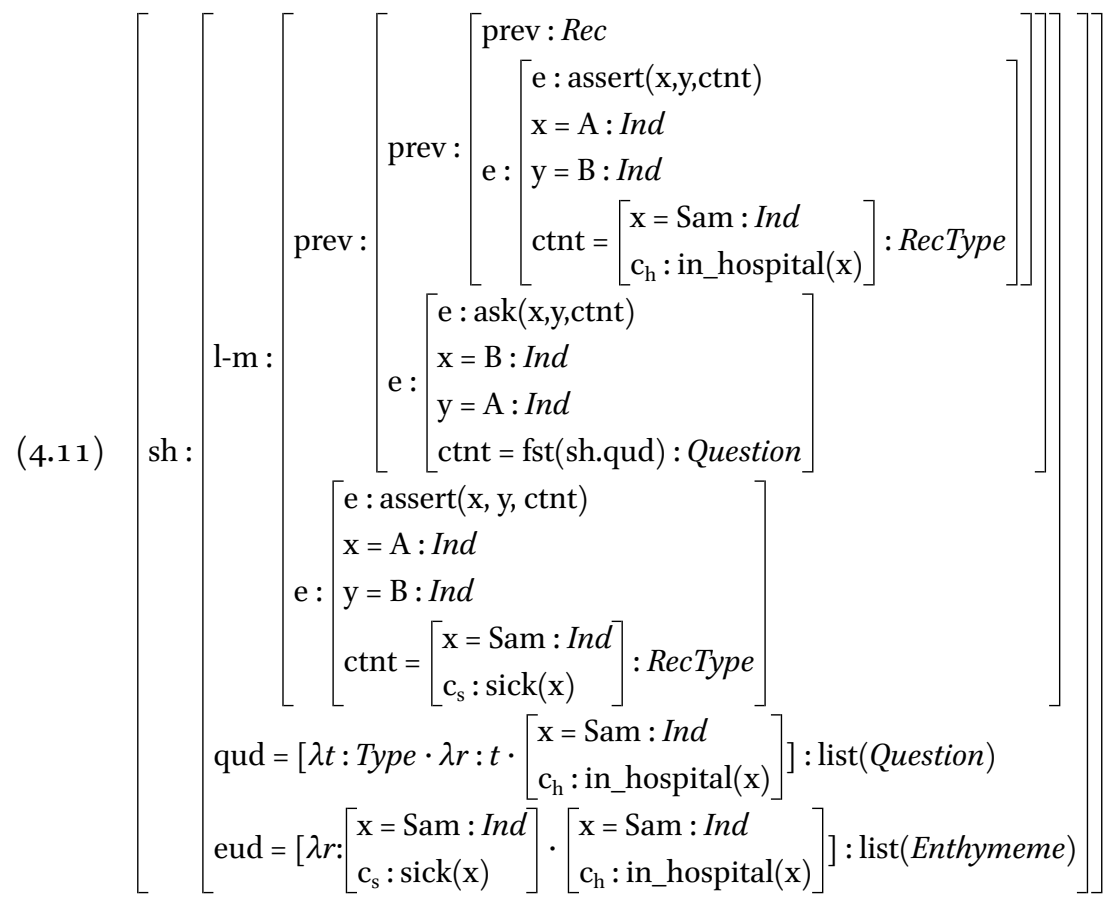

\subsection{Coordinating on Topoi}

In the previous section (4.1), we have looked at how an enthymeme under discussion is integrated on the dialogue gameboard as the result of a Whyquestion. In this section, we will look at various ways in which dialogue participants can coordinate on the topoi underpinning enthymemes in dialogue.

\subsubsection{Integrating Topos}

Let us return to our example (repeated here for convenience).

(4.12) a. A: Sam's in hospital.

b. $B$ : Why?

c. $A$ : He's sick.

The enthymeme "Sam is in the hospital since he is sick" is now part of B's information state, meaning that he has interpreted (4.12c) as given by $A$ as a reason for the content of utterance (4.12a). There are (at least) two goals associated with the utterance (4.12c): First, $A$ wants $B$ to recognise the reason given as something which is relevant in relation to (4.12a). Second, she wants $B$ to accept 
it as an explanation or support of the assertion (4.12a). For the first goal to be achieved, $B$ must have access to a topos warranting the enthymeme. In other words: The interpretation of an enthymeme by an agent is much facilitated if the agent has access to a warranting principle which is more generally applicable than the enthymeme conveyed in the discourse. Consider the following example:
(4.13) a. A: I am hungry.
b. $B$ : Have a piece of fruit!

The type of situation where the speaker is hungry is a subtype of a type of situation where someone is hungry. Also, a situation where the speaker is having a piece of fruit is a subtype of a type of situation where someone eats something. Thus, a topos that provides a warrant for the dialogue in (4.13) could be somethig like "if you are hungry, you should eat something".

Likewise, the enthymeme "Sam is sick, therefore he is in hospital" is underpinned by a topos associating being sick with being in or going to hospital. In (4.14a) we see the enthymeme "Since Sam is sick, he is in hospital". In (4.14b) we see the topos drawn on to interpret the enthymeme ("İf someone is sick, they are in hospital").
a. $\lambda r:\left[\begin{array}{l}\mathrm{x}=\operatorname{Sam}: \text { Ind } \\ \mathrm{c}_{\mathrm{s}}: \operatorname{sick}(\mathrm{x})\end{array}\right] \cdot\left[\mathrm{c}_{\mathrm{h}}:\right.$ in_hospital $\left.(r \cdot \mathrm{x})\right]$
b. $\lambda r:\left[\begin{array}{l}\mathrm{x}: \text { Ind } \\ \mathrm{c}_{\mathrm{s}}: \operatorname{sick}(\mathrm{x})\end{array}\right] \cdot\left[\mathrm{c}_{\mathrm{h}}:\right.$ in_hospital $\left.(r . \mathrm{x})\right]$

A key idea in our theory is that enthymemes conveyed in discourse are often specifications of more generally applicable topoi. For example, the utterance "Kitty likes milk, she is a cat" conveys the enthymeme "Kitty is a cat, therefore she likes milk". This enthymeme is a specification of a topos saying that if $x$ is a cat, then $x$ likes milk. In this case the specification amounts to the topos being restricted to concern just the individual Kitty, but a specification could also involve adding constraints to the type of situation to which an enthymeme or a topos applies, the antecedent- or domain type of the function.

In the formal account, the notion of specification is linked to subtyping (see Section 3.2.2). The requirements for an enthymeme $\varepsilon$ to be a specification of a topos $\tau$, is that the antecedent type of $\varepsilon$ must be a subtype of the antecedent type of $\tau$, and the result of applying $\varepsilon$ to any record (representing, for example, a situation) must be a subtype of the result of applying $\tau$ to the same record. 
(4.15) Assuming topos $\tau=\lambda r: \mathrm{T}_{1} \cdot T_{2}$ and enthymeme $\varepsilon=\lambda r: \mathrm{T}_{3} \cdot T_{4}, \varepsilon$ is a specification of $\tau$, i.e., specification $(\varepsilon, \tau)$ is witnessed, iff $T_{3} \sqsubseteq T_{1}$ and for any $r, \varepsilon(r) \sqsubseteq \tau(r)$.

It should be emphasised that the perceived relation between enthymemes and topoi is relative to the agents involved in dialogue-dialogue participants can disagree about whether a given enthymeme is a specification of a particular topos. For an enthymematic dialogue contribution to be interpreted correctly, it is important that the enthymeme is recognisable as a specification of a topos for that topos to be evoked in the discourse. From a rhetorical point of view, to maximise persuasion, the topos must also be acceptable to the audience in the context where it is being evoked.

When interpreting an enthymeme, an agent compares the topos they have on private on the DGB to the enthymeme. If the agent recognises the enthymeme as a specification of the private topos, the topos is loaded onto the shared DGB as the topos believed by the interpreting agent to underpin the enthymeme. In (4.16), we see the update rule $f_{\text {integrate_topos }}$. When applied to an information state including both a private topos and an enthymeme which is a specification of that topos, ${ }^{1}$ it returns a type where the private topos has been integrated in the shared DGB.

$$
\begin{aligned}
& f_{\text {integrate_topos }}= \\
& \lambda r:\left[\begin{array}{l}
\text { private }:[\text { topoi }: \operatorname{list}(\text { Topos })] \\
\text { shared }:\left[\begin{array}{l}
\text { eud }: \operatorname{list}(\text { Enthymeme }) \\
\text { topoi }: \operatorname{list}(\text { Topos })
\end{array}\right]
\end{array}\right] . \\
& \lambda e:\left[\begin{array}{l}
\mathrm{t}: \text { Topos } \\
\mathrm{c}_{1}: \text { in }(\mathrm{t}, r \text {.private.topoi }) \\
\mathrm{c}_{2}: \text { specification }(\mathrm{fst}(r . \text { shared.eud }), \mathrm{t})
\end{array}\right] . \\
& \quad[\text { shared }:[\text { topoi }=[\text { e.t } \mid r \text { r.shared.topoi }]: \operatorname{list}(\text { Topos })]]
\end{aligned}
$$

We will call the type of $B$ 's information state before the update of shared topos $T_{4}$.

(4.17) $T_{4} \sqsubseteq\left[\operatorname{pr}:\left[\right.\right.$ topoi $=\left[\lambda r:\left[\begin{array}{l}\mathrm{x}: \text { Ind } \\ \mathrm{c}_{\mathrm{s}}: \operatorname{sick}(\mathrm{x})\end{array}\right] \cdot\left[\mathrm{c}_{\mathrm{h}}: \operatorname{in} \_\right.\right.$hospital$\left.\left.(\mathrm{r.x})\right]\right]: \operatorname{list}($ Topos $\left.\left.)\right]\right]$

1 We use abstraction over $e$ to introduce conditions on information states whose witnesses are not part of the information state proper. 
The shared part of $T_{4}$ is identical to that in (4.11). To update 'shared topos', we apply $f_{\text {integrate_topos }}$ to an information state $s_{4}$ of type $T_{4}$, and a record that witnesses that the first enthymeme in 'shared.eud' is a specification of a topos in 'private.topoi', resulting in a type $T_{5}$ shown in (4.18).

(4.18) $T_{5}=\left[\operatorname{sh}:\left[\right.\right.$ topoi $=\left[\lambda r:\left[\begin{array}{l}\mathrm{x}: \text { Ind } \\ \mathrm{c}_{\mathrm{s}}: \operatorname{sick}(\mathrm{x})\end{array}\right] \cdot\left[\mathrm{c}_{\mathrm{h}}: \operatorname{in} \_\right.\right.$hospital $\left.\left.(r \cdot \mathrm{x})\right]\right]: \operatorname{list}($ Topos $\left.\left.)\right]\right]$

After having asymmetrically merged $T_{4}$ with $T_{5}$ we get the type of the updated information state, seen in (4.19).

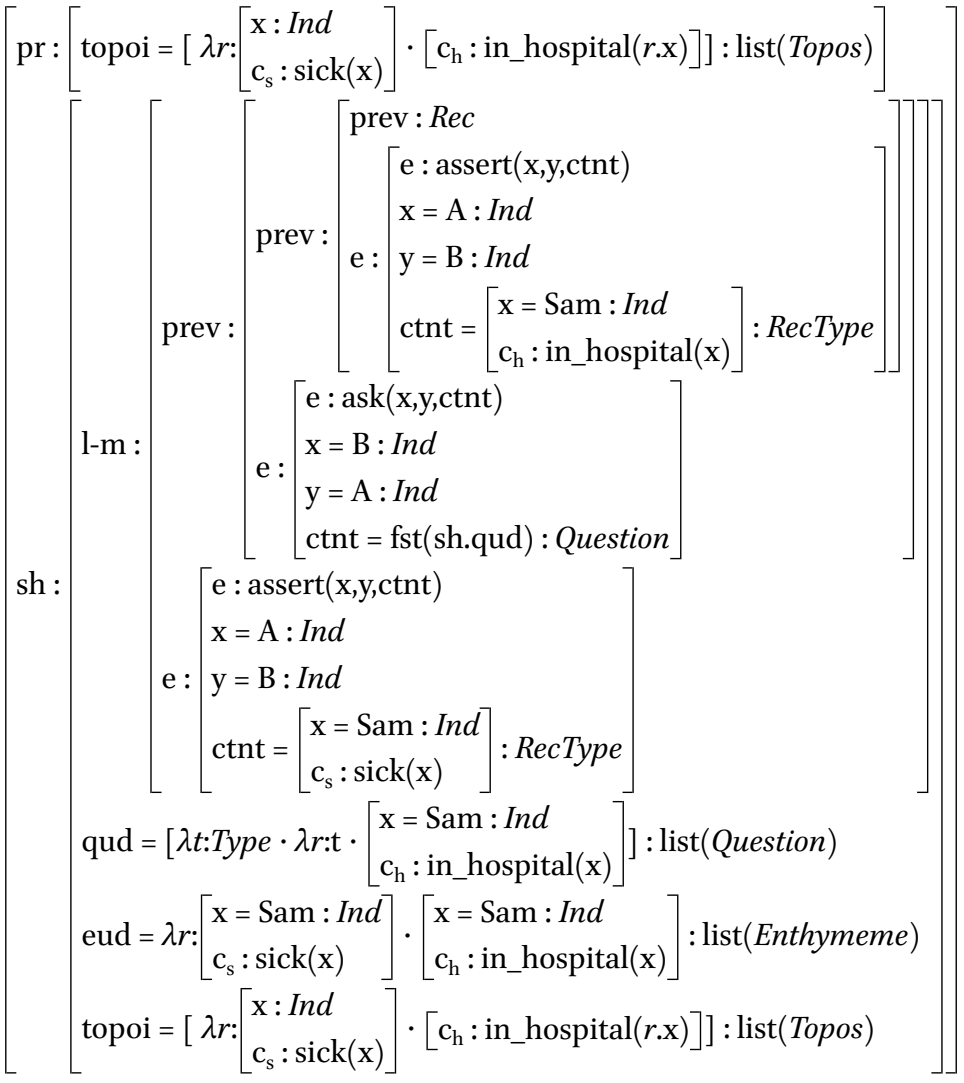

4.2.1.1 Understanding and Accepting Arguments

The update rule just presented, $f_{\text {integrate_topos }}$, represents the process of an agent recognising a topos and assuming that it is indeed this topos on which the speaker is basing her argument. It should be emphasised that this does not necessarily mean that the agent agrees with or accepts the assertion made (the 
conclusion of the argument), but merely that she recognises it as underpinning the enthymeme.

There is a difference between interpreting an enthymeme in a coherent way and agreeing to the claim or proposition made, as illustrated by the example in (4.20):

\author{
(4.20) a. Child: Can I go swimming? \\ b. Father: No, we just had lunch. \\ c. Child: But dad, swimming after meals is actually not harmful!
}

The child in the previous example accommodates a topos presumably underpinning the father's argument, namely the notion that it is dangerous to swim after meals (which research has shown to be false). In order to correctly interpret the enthymeme the child must be familiar with this notion. However, this does not mean that she agrees with it. The two levels of interpretation of enthymemes we will focus on here are understanding and acceptance. By the former we mean that the agent in question has access to a relevant topos, by the latter that the interlocutor accepts the enthymeme and tentatively accommodates a topos based on the enthymeme. However, none of these actually mean that the interpreting agent agrees with the conclusion or that she agrees that the topos holds and is relevant in the context. Still, as is the case with presupposition accommodation, if the dialogue continues after the accommodation of a topos without any questioning from the interlocutor, we can only assume that the interlocutor agrees, at least for the time being. Obviously, issues, questions, enthymemes and topoi may be raised and reraised at any time. Those points in discourse where reraising occurs are often interesting, as topoi are often made explicit in such contexts. This also gives the interlocutors an opportunity to coordinate to make sure that they draw on the same topos when interpreting an enthymeme. In the next section we will look further at what happens when interlocutors draw on different topoi to underpin enthymemes in the discourse, and how they resolve such situations.

\title{
4.2.2 Topoifrom Underspecified Enthymemes
}

In the previous section we considered how a why-question can be seen as eliciting an enthymeme. If this enthymeme matches a topos available to and accepted by the agent posing the question, chances are that he or she will be satisfied with the reply. Arguably, the assumption in such cases is that the underpinning of the argument, the topos, is similar in the information states 
of the conversational participants. However, it is also possible that two agents involved in dialogue accommodate different topoi which both satisfy the criteria for underpinning a particular enthymeme. This kind of mismatch of topoi may go unnoticed in cases where consensus is reached. After all, if interlocutors agree, there is often no reason to argue about the rationale for agreeing.

In this section, we will look at a dialogue example where it is made explicit that the speaker and the listener interpret an enthymeme drawing on different topoi. This snippet of dialogue is taken from a radio program where discussion alternates with music. The interviewee is Swedish hip hop artist Petter, and much of the dialogue relates to the songs being played in the music sections. Just before the dialogue in (4.21) a tune by a metal band has been played. Petter is being asked for his opinion of the song.
(4.21) a. $P$ : Metal was actually the reason I started doing hip hop
b. $P$ : ... because I hated metal
c. $J$ : Oh, I thought you were going to say something completely differ- ent!

This example is interesting since it provides evidence that dialogue participant $J$ reasons incrementally about the enthymeme $P$ is producing. In fact, $P$ 's utterance can be seen as an incomplete enthymeme - there is something about metal that made Petter start doing hip hop. We think of this as the antecedent type of the enthymeme being underspecified. Intuitively, this means that there is no readily available topos warranting this enthymeme. ${ }^{2}$ We can think of the utterance in (4.21a) as describing a situation where the music genre metal occurs, leading to a situation where the speaker starts practicing music of the hip hop genre. Thus, the enthymeme is a function of the type seen in (4.22). We refer to this enthymeme as $\varepsilon_{\text {reason }}$.

(4.22) $\varepsilon_{\text {reason }}=\lambda r:\left[\begin{array}{l}\mathrm{T}=\text { Music }: \text { Type } \\ \mathrm{x}=\text { metal }: \mathrm{T} \\ \mathrm{z}=\text { Petter }: \text { Ind } \\ \mathrm{c}_{1}: \text { relevant }(\mathrm{T})\end{array}\right] \cdot\left[\begin{array}{l}\mathrm{y}=\text { hiphop }: r . \mathrm{T} \\ \mathrm{c}_{2}: \operatorname{do}(r . \mathrm{z}, \mathrm{y})\end{array}\right]$

2 As pointed out previously regarding the relation between enthymeme and topos, underspecification is context relative. It is not inconceivable that there would be a topos warranting this enthymeme in a particular context. 
There might be several topoi accessible to $J$ which could be drawn on to underpin the enthymeme in (4.22). Judging from $J$ 's utterance she is surprised by $P$ 's assertion that he hated metal. We cannot say exactly in which way Petter hating metal is "completely different" from what $J$ expected. However, it seems reasonable to assume that she expected metal being the reason for $P$ starting to "do" hiphop being due to some positive quality of metal or some positive relation between him and metal. Thus, a possible topos could be one saying that if two things are of the same type, and something positive is associated with one of them, that thing may cause someone to "do" the other thing. From a logical point of view this principle is, of course, shaky at best. However, it still seems to be fairly productive in everyday argumentation. Think of examples like "Karate got me interested in Kung Fu", etc. We see a formalisation of this topos, $\tau_{\text {similar }}$ in (4.23).

(4.23) $\tau_{\text {similar }}=\lambda r:\left[\begin{array}{l}\mathrm{T}: \text { Type } \\ \mathrm{x}: \mathrm{T} \\ \mathrm{z}: \text { Ind } \\ \mathrm{c}_{1}: \operatorname{relevant}(\mathrm{T}) \\ \mathrm{c}_{2}: \operatorname{like}(\mathrm{z}, \mathrm{x})\end{array}\right] \cdot\left[\begin{array}{l}\mathrm{y}: r \cdot \mathrm{T} \\ \mathrm{c}_{3}: \operatorname{do}(r \cdot \mathrm{z}, \mathrm{y})\end{array}\right]$

In the previous section we introduced the rule integrate topos, (4.16), for adding topoi to the shared field of the DGB. According to this update rule, a particular enthymeme justifies the loading of a topos onto the DGB if the enthymeme is a specification of the topos. We defined that as meeting two conditions. First, the domain- or antecedent part of the enthymeme must be a subtype of (i.e., more specific or identical to) the corresponding part of the topos. Secondly, the result of applying the enthymeme to a record $r$ must be a subtype of the result of applying the topos to the same record.

If we compare $\tau_{\text {similar }}$ and $\varepsilon_{\text {reason }}$, we see that the domain type of $\varepsilon_{\text {reason }}$ is not a subtype of the domain type of $\tau_{\text {similar }}$, since it lacks the constraint $\mathrm{c}_{2}$ : $\operatorname{like}(\mathrm{z}, \mathrm{x})$. The first requirement for applying the update function $f_{\text {integrate_topos }}($ see $(4.16))$ is thus not met.

However, since dialogue participants sometimes do accommodate topoi based on underspecified enthymemes, we want to be able to model how topoi may be integrated based on less strict requirements. In order to do this we introduce an additional update rule $f_{\text {integrate_topos' }}$. This rule says that we may integrate a topos on the shared gameboard if the application of the first enthymeme on the list of enthymemes under discussion (we may call this max-EUD, parallel to Ginzburg's (2012) terminology for QUD) to any $r$, is a subtype of the application of the topos to $r$, and there is no accessible topos where the rule $f_{\text {integrate_topos }}$ 
applies. In other words: if there is no accessible topos that is a more general version of the enthymeme, look for a topos of which the enthymeme is a more general version. We talk about this kind of enthymeme as underspecified in relation to the topos. In (4.24) is a more precise definition of the concept of an underspecified enthymeme.

(4.24) Assuming topos $\tau=\lambda r: \mathrm{T}_{1} \cdot T_{2}$ and enthymeme $\varepsilon=\lambda r: \mathrm{T}_{3} \cdot T_{4}, \varepsilon$ is an underspecification of $\tau$, i.e., underspecification $(\varepsilon, \tau)$ is witnessed, iff $T_{1} \sqsubset T_{3}$ and for any $r, \varepsilon(r) \sqsubseteq \tau(r)$.

Since the domain type of $\varepsilon_{\text {reason }}$ includes manifest fields which are not manifest in the domain type of $\tau_{\text {similar }}, \varepsilon_{\text {reason }}$ is not underspecified with regard to $\tau_{\text {similar }}$. However, we are always allowed to generalise enthymemes by removing the values of manifest fields (making manifest fields non-manifest) in the domain type. We may also remove entire fields of the domain type as long as nothing in the result type depends on the removed field.

If we generalise $\varepsilon_{\text {reason }}$ by removing the values of $\mathrm{x}$ and $\mathrm{z}$, we obtain the enthymeme in (4.25):

(4.25) $\varepsilon_{\text {reason } n_{\text {gen }}}=\lambda r:\left[\begin{array}{l}\mathrm{T}: \text { Type } \\ \mathrm{x}: \mathrm{T} \\ \mathrm{z}: \text { Ind } \\ \mathrm{c}_{1}: \operatorname{relevant}(\mathrm{T})\end{array}\right] \cdot\left[\begin{array}{l}\mathrm{y}=\text { hiphop }: r . \mathrm{T} \\ \mathrm{c}_{2}: \mathrm{do}(r . \mathrm{z}, \mathrm{y})\end{array}\right]$

We may now finally apply the rule for integrating topoi on the shared DGB based on an underspecified enthymeme- $f_{\text {integrate_topos' }}$.

(4.26) $f_{\text {integrate_topos' }}=$

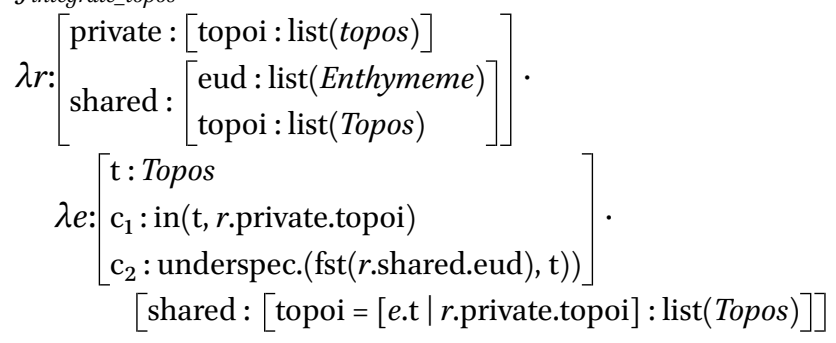

$\tau_{\text {similar }}$ is on J's private gameboard at the start of the operation to integrate it on her shared gameboard. For now, let us not care about exactly how this topos got selected to the private gameboard, let us just assume that the private gameboard holds a depository of topoi that the speaker finds salient in the context. 
Furthermore, we assume that we have $s_{1}$ representing J's information state, and $e_{1}$ imposing conditions as in (4.27):

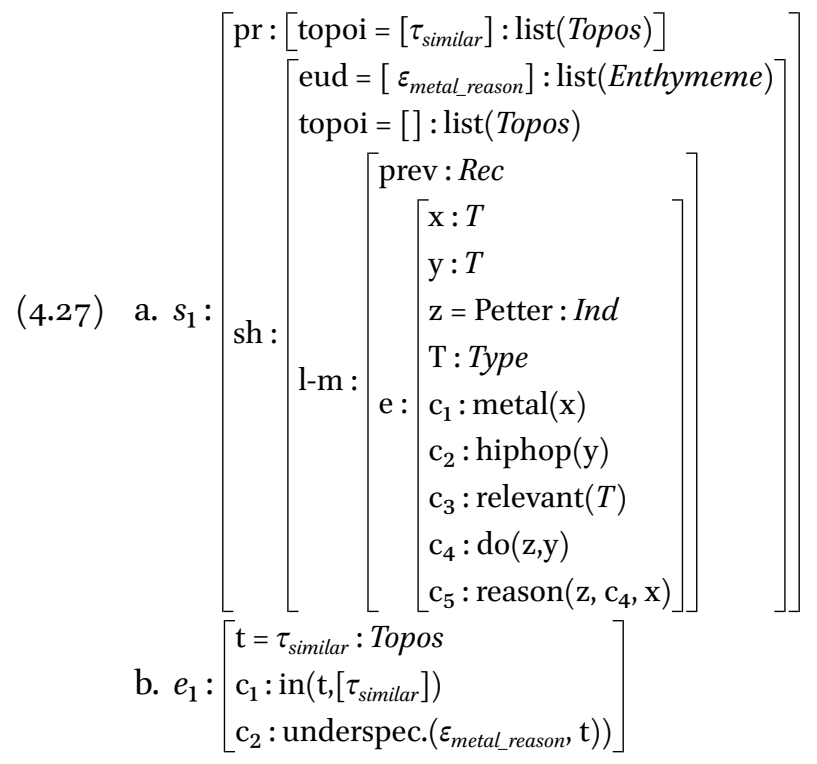

(4.28) $f_{\text {integrate_topos' }}\left(s_{1}\right)\left(e_{1}\right)=$

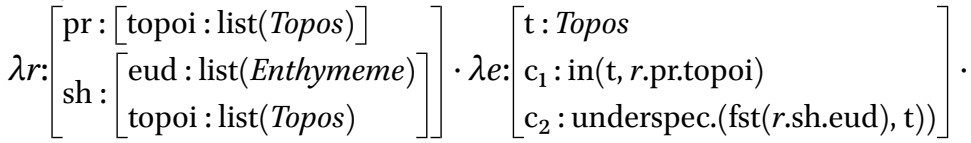

$$
\begin{aligned}
& {[\text { sh }:[\text { topoi }=[\text { e.t } \mid r \text {.private.topoi }]: \operatorname{list}(\text { Topos })]]\left(r_{1}\right)\left(e_{1}\right)=} \\
& {\left[\text { sh }:\left[\text { topoi }=\left[\tau_{\text {similar }}\right]: \operatorname{list}(\text { Topos })\right]\right]}
\end{aligned}
$$


To obtain the type of the updated information state for $J$, we do an asymmetric merge of the type of $r_{1}$ and the result type, as seen in (4.29).

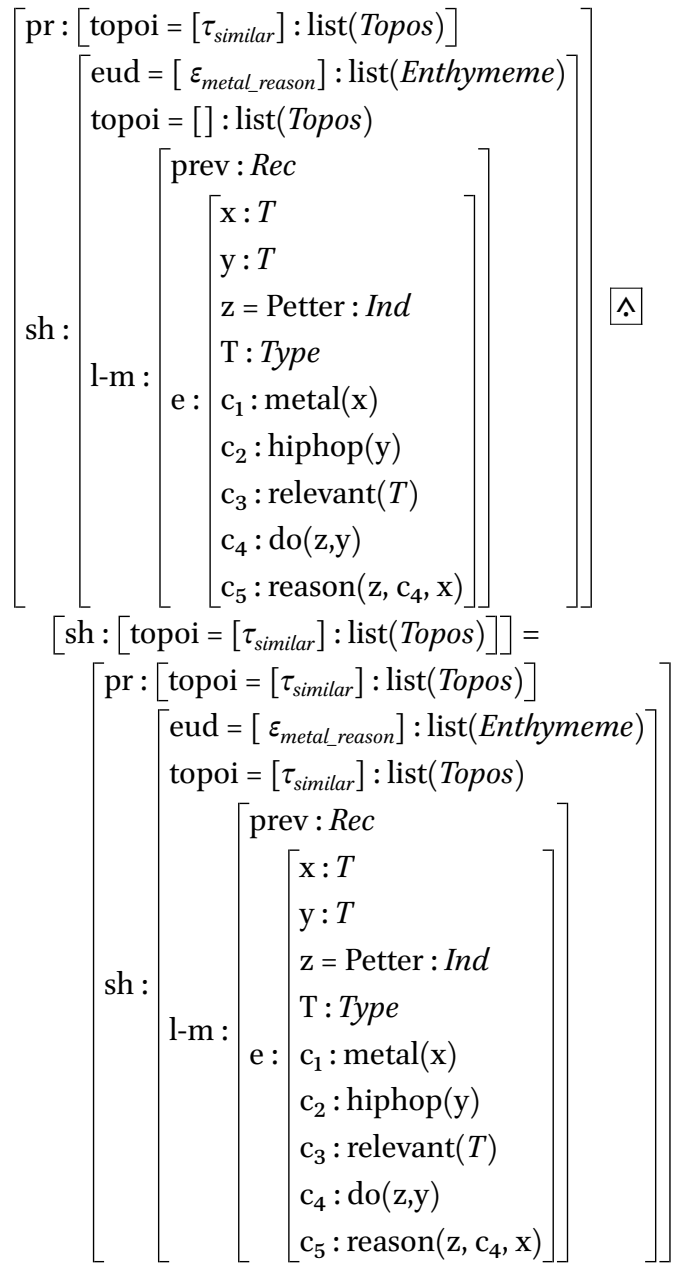

This update of J's information state reflects that she is reasoning incrementally, and has a preconception about $P$ 's reasoning based on what he has said this far. In situations where dialogue participants know each other very well and/or the context allows it, participants may well infer topoi based on underspecified enthymemes, which turn out to be exactly the ones intended by the speaker. Furthermore the possibility of asking follow-up questions and other types of feedback make it efficient to reason based on underspecified enthymemes in situations where the stakes are not too high. 
To summarise this section so far, we may say that when trying to interpret an enthymeme, a dialogue participant first tries to access a topos that serves as underpinning for the enthymeme. Most obviously this means a topos which is a bit more general than the enthymeme. However, if no such topos is accessible to the dialogue participant, he may tentatively integrate another topos accessible on his private gameboard, if this topos is similar to-but actually more specific than-the enthymeme under discussion since the antecedent part involves more constraints.

\subsubsection{Re-raising Topoi}

When we engage in conversation we normally try to interpret underspecified or implicit content drawing on information already introduced on the shared DGB. This is the case with for example resolution of anaphora. Thus we would want an algorithm for applying update rules meant to pick out a topos to underpin the enthymeme currently under discussion, to first apply the rule $f_{\text {reraise_topos }}$ which looks for a suitable topos which is already part of common ground, that is already present on the DGB, and not until that fails, apply the rules described in Sections 4.2.1 and 4.2.2 which look into the conversational participant's private depository of salient topoi.

The rule in (4.30) says that if the information state has a topos on 'shared.topoi' supporting the max-EUD (the most recently conveyed enthymeme), we are licensed to update that information state so that the topos in question is moved to the top of the list of topoi.

In (4.30), we use a function $\mu$. If $b$ is a list and $a \in b$, the function $\mu$ applied to $b, \mu(a, b)$, moves $a$ to the top of list $b$ regardless of what position $a$ has had previously. The use of ordered lists for topoi present on the shared DG B captures some aspects of salience. When a new topos is added it is always placed at the top of the list. This entails that the list of topoi is connected to the chronological order in which the topoi were added to the discourse model. However, this order may change if a topos which is already integrated on shared topoi is reraised in the conversation.

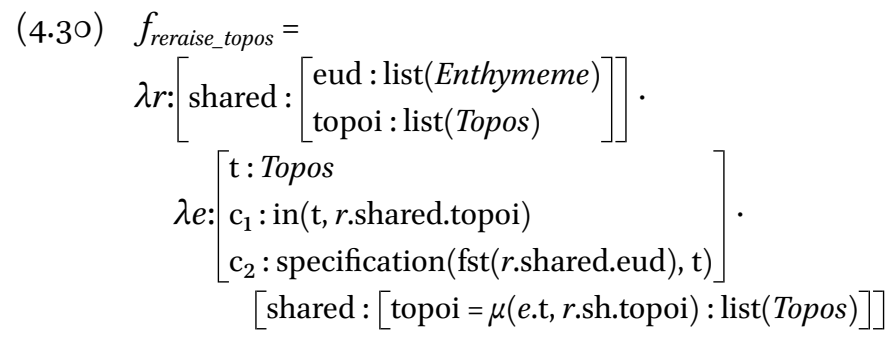




\subsubsection{Re-evaluating Topoi}

In everyday conversational settings we tend to make inferences without having, in a logical sense, adequate evidence. For this reason dialogue participants sometimes need to reevaluate the principles on which they base their interpretations of enthymemes.

As we move on to the final part of Petter's utterance, (4.21b), we are faced with precisely this problem - the enthymeme which is now first on the list of enthymemes under discussion does not match the topos first on the list of shared topoi. The reason for this is that when integrating (4.21a), $J$ integrates the enthymeme $\varepsilon_{\text {reason }}$ in (4.31a), and accommodates a topos underpinning this enthymeme, $\tau_{\text {similar }}$. When encountering (4.21b), $J$ learns that in fact $P$ had a different enthymeme in mind, $\varepsilon_{\text {reason }}$ in (4.31b). The intuition here is that the new enthymeme requires a new topos to be added to the DGB.

$$
\begin{aligned}
& \text { a. } \varepsilon_{\text {reason }}=\lambda r:\left[\begin{array}{l}
\mathrm{T}=\text { music }: \text { Type } \\
\mathrm{x}=\text { metal }: \mathrm{T} \\
\mathrm{z}=\text { Petter }: \text { Ind } \\
\mathrm{c}_{1}: \operatorname{rrlevant}(\mathrm{T})
\end{array}\right] \cdot\left[\begin{array}{l}
\mathrm{y}=\text { hiphop }: r . \mathrm{T} \\
\mathrm{c}_{2}: \operatorname{do}(r . \mathrm{z}, \mathrm{y})
\end{array}\right] \\
& \text { b. } \varepsilon_{\text {reason' }}=\lambda r:\left[\begin{array}{l}
\mathrm{T}: \text { Type } \\
\mathrm{x}=\text { metal }: \mathrm{T} \\
\mathrm{c}_{1}: \operatorname{relevant}(\mathrm{T}) \\
\mathrm{z}=\text { Petter }: \text { Ind } \\
\mathrm{c}_{\text {hate }}: \text { hate }(\mathrm{z}, \mathrm{x})
\end{array}\right] \cdot\left[\begin{array}{l}
\mathrm{y}=\text { hiphop }: r . \mathrm{T} \\
\mathrm{c}_{2}: \operatorname{do}(r . \mathrm{z}, \mathrm{y})
\end{array}\right]
\end{aligned}
$$

The only difference between the two enthymemes is that the antecedent type of $\varepsilon_{\text {reason' }}$ includes one constraint, $\mathrm{c}_{\text {hate }}$, which is not present in $\varepsilon_{\text {reason }} \cdot \varepsilon_{\text {reason }}$, is thus a specification of $\varepsilon_{\text {reason }}$.

In 4.2.2 we discussed what it means for an enthymeme to be a specification of a topos and what it means for an enthymeme to be underspecified in relation to a topos. There are situations - such as the present one of $J$ trying to make sense of $P$ 's utterance-where the enthymeme seems to be incompatible with the topos at the top of the list of 'sh.topos' (max-topos). Here the enthymeme $\varepsilon_{\text {reason' }}$ says that since $P$ hated metal, he started doing hip hop, and the topos $\tau_{\text {similar }}$ says that if someone likes something that person might also get involved in other, similar, activities. The antecedents of $\varepsilon_{\text {reason' }}$ and $\tau_{\text {similar }}$ include concepts that we would probably want to model as mutually exclusive, namely like and hate. The formula in (4.32) is our version of a meaning postulate, and reads " $T_{1}$ precludes $T_{2}$ ", that is there is no situation which is both of type $T_{1}$ and of type $T_{2}$. 
(4.32) If $\left[\begin{array}{l}\mathrm{x}: \text { Ind } \\ \mathrm{c}: \text { hate }(\mathrm{x})\end{array}\right]=T_{1}$ and $\left[\begin{array}{l}\mathrm{x}: \text { Ind } \\ \mathrm{c}: \operatorname{like}(\mathrm{x})\end{array}\right]=T_{2}$ then $T_{1} \perp T_{2}$

The only topos on the list of shared topoi at the point where $J$ has just integrated $\varepsilon_{\text {reason }}$ is such that the max-EUD cannot be a specification of it, nor can the topos be a specification of the max-EUD, since $\varepsilon_{-}$reason' $\perp \tau_{\text {similar }}$. Thus the conditions for applying $f_{\text {reraise_topos }}$ are not fulfilled. So, we move on to once again applying rule $f_{\text {integrate_topos. }}$ A topos that would work here would be one capturing the notion of "the lesser of two evils", $\tau_{l_{-} t_{-} e}$. The idea of this topos is that if, in a particular context or situation, you have two things to choose from and one is bad, you pick the other one.

(4.33) $\tau_{l \_t \_e}=\lambda r:\left[\begin{array}{l}\mathrm{T}: \text { Type } \\ \mathrm{x}: \mathrm{T} \\ \mathrm{y}: \mathrm{T} \\ \mathrm{z}: \text { Ind } \\ \mathrm{c}_{1}: \operatorname{relevant}(\mathrm{T}) \\ \mathrm{c}_{2}: \operatorname{hate}(\mathrm{z}, \mathrm{y})\end{array}\right] \cdot\left[\mathrm{e}: \operatorname{start} \_d o i n g(r . z, r . \mathrm{x})\right]$

We assume thus, that J's information state when she has integrated $\varepsilon_{\text {reason }}$, is of the type in (4.34).

(4.34) $T_{J} \sqsubset\left[\begin{array}{l}\left.\text { private : }\left[\begin{array}{l}\text { topoi }=\left[\tau_{\iota_{\perp} \_}\right] \\ \end{array}\right]: \operatorname{list}(\text { Topos })\right] \\ \text { shared : }\left[\begin{array}{l}\text { eud }=\left[\varepsilon_{\text {reason }}{ }^{\prime}, \varepsilon_{\text {reason }}\right]: \operatorname{list}(\text { Enthymeme }) \\ \text { topoi }=\left[\tau_{\text {similar }}\right]: \operatorname{list}(\text { Topos })\end{array}\right]\end{array}\right]$

Since the application of update rule $f_{\text {reraise_topos }}$ fails in this situation, we move on to apply $f_{\text {integrate_topos }}$ to the information state $s_{J}$ of type $T_{J}$.

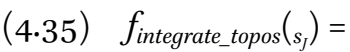

$\left[\right.$ shared : $\left[\begin{array}{l}\text { eud }=\left[\varepsilon_{\text {reason }}, \varepsilon_{\text {reason }}\right]: \operatorname{list}(\text { Enthymeme }) \\ \text { topoi }=\left[\tau_{l_{-} \_e}, \tau_{\text {similar }}\right]: \operatorname{list}(\text { Topos })\end{array}\right]$

\subsubsection{Accommodating Resource Topoi}

In his classic paper Scorekeeping in a language game, Lewis (1979) argues that if you "say something that requires a missing presupposition, that presupposition will straight away spring into existence". Lewis refers to this phenomenon as accommodation (See also Karttunen, 1974 and Stalnaker, 1974).

When a speaker makes an utterance like that in (4.36), the claim-that the rollers must be carefully looked after since they are of much higher quality com- 
pared to the kind that is normally used - presupposes a topos warranting high quality as a reason for the rollers being well looked after. This topos could be something along the lines of "if something is valuable, it should be well looked after". The topos must, if we accept the enthymeme conveyed, be integrated on the shared DGB. We may refer to this integration as a kind of accommodation.

(4.36) A: I'm going to take a, a roller 〈pause $\rangle$ these are very expensive, very classy rollers.

$A$ : $\langle$ cough $\rangle$ Much higher quality than the bioprinting [sic] rollers that we may be used to using.

$A$ : And therefore they must be carefully looked after.

(BNC: F77 341-343)

However, in the case of presuppositions, it is clear from the triggering sentence exactly what is presupposed. In the case of an accommodated topos, it is clear only that some topos is drawn on to warrant the enthymeme in the discourse. Above, we have considered two ways of updating shared topoi on the DGB$f_{\text {integrate_topos }}(4.16)$ and $f_{\text {integrate_topos' }}$ (4.26), which move a topos from an agent's private DGB and integrate it on the shared DGB-and $f_{\text {reraise_topos }}$ which finds an appropriate topos on the shared DG B and moves it to the front of the list of shared topoi.

In addition to the types of accommodation mentioned, there are other possible types of topos accommodation. First, adding a topos to the shared DG B which is not part of the depository of private salient topoi (corresponding to the list on 'private.topoi') but must be retrieved from long term memory (we refer to this depository as the agent's rhetorical resources). Another type of accommodation is when a conversational participant does not have access to a topos which matches the enthymeme currently under discussion, and tentatively construes one based on the enthymeme under discussion.

In a situation such as (4.36), if a dialogue participant is not aware of the principle "costly/high quality things should be taken care of", he might integrate a topos such as "if a roller is expensive it should be taken care of" or, more generally, "if an artefact is expensive it should be taken care of". This topos may then be tentatively added to the resources of the individual, and eventuallyif reinforced in further interaction-be considered a reliable topos.

The first of these scenarios, where a topos is available in the long term memory of the dialogue participant (shown in (4.37)), we refer to as $f_{\text {integrate_resource_topos. }}$. 
(4.37) $f_{\text {integrate_resource_topos }}=$

$$
\begin{aligned}
\lambda r:\left[\begin{array}{l}
\text { private :[topoi }: \operatorname{list}(\text { topos })] \\
\text { shared }:\left[\begin{array}{l}
\text { eud }: \operatorname{list}(\text { Enthymeme }) \\
\text { topoi }: \operatorname{list}(\text { Topos })
\end{array}\right]
\end{array}\right] . \\
\lambda e:\left[\begin{array}{l}
\mathrm{t}: \text { Topos } \\
\mathrm{c}_{1}: \text { in_rhet_resources }(\mathrm{t}) \\
\mathrm{c}_{2}: \text { specification }(\mathrm{fst}(r \text {.shared.eud }), \mathrm{t})
\end{array}\right] \cdot \\
\quad[\text { shared }:[\operatorname{topoi}=[\text { e.t } \mid r \text {.shared.topoi }]: \operatorname{list}(\text { Topos })]]
\end{aligned}
$$

We also need a rule to account for cases where the enthymeme is underspecified. We see such a rule in (4.38).

$$
\begin{aligned}
& \text { (4.38) } f_{\text {integrate_resource_topos' }}=
\end{aligned}
$$

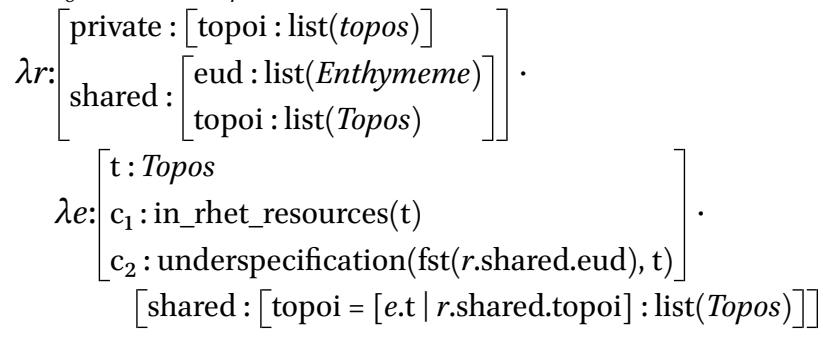

In all of the cases above we would say that accommodation occurs, if we take accommodation to mean integration of a pragmatic inference on the shared DGB. However, in the scenario above, where a dialogue participant tentatively adds a topos to the gameboard based on the enthymeme under discussion, we not only accommodate a topos, we also add something to our resources that was not there before. This kind of topos accommodation will be discussed further in Chapters 5 and 6.

\subsection{Summary}

In this chapter we first looked at an example where the enthymematic structure is made explicit by a why-question. We considered how why-questions relate to enthymemes and how answers to questions can be evaluated based on how the enthymemes conveyed relate to accessible topoi.

We also considered an example where dialogue participants interpret the same enthymeme drawing on different topoi. To accommodate this type of dialogue event we extended our set of rules. We also had to make adjustments to the order in which rules are applied, i.e. the update algorithm. 
One reason that dialogues can develop in the way described above is that speakers use enthymemes which are underspecified in relation to the topos drawn on. In our case the first enthymeme is underspecified in relation to the topos based on which $J$ interprets it, as well as in relation to the topos that $P$ seems to have in mind. It may well be argued that there is a preference for the first interpretation, and $P$ is thus being deliberately misleading in first presenting an underspecified enthymeme. Note that we have defined underspecified enthymemes, but which topoi that may be conceived of as appropriate underpinning for any particular enthymeme is an empirical question. It is possible that an enthymeme which is underspecified according to our definition, would easily convey the intended enthymeme in a particular context, and vice versa.

We have focused on the issue of underspecified enthymemes and how they can still be used to accommodate particular topoi. However, in the discussion of evaluation of topoi in relation to the enthymeme under discussion, we made some simplifying assumptions. For example, we might want to introduce rules that remove enthymemes which include premises which are incompatible with more recently added enthymemes. Some of these issues will be addressed in the next two chapters. 